\title{
Acute hepatic encephalopathy and multiorgan failure in sickle cell disease and COVID-19
}

\author{
Giulia Martone ${ }^{1}$, Priyanka Nanjireddy ${ }^{1}$, Robin Craig $^{1}$, Andrew Prout ${ }^{1}$, Meghan Higman ${ }^{1}$, \\ Kara Kelly ${ }^{1}$, Steven Ambrusko ${ }^{1}$, and Alberto Pepe ${ }^{2}$ \\ ${ }^{1}$ University at Buffalo Jacobs School of Medicine and Biomedical Sciences \\ ${ }^{2}$ Authorea Team
}

June 11, 2020

\begin{abstract}
COVID-19 disease causes primarily pulmonary manifestations, with myriad other clinical manifestations especially in high-risk conditions, including sickle cell disease (SCD). We present a 19-year-old with SCD on deferasirox with COVID-19 infection involving pain and acute chest syndrome, four weeks later developing hyperammonemia and hyperinflammatory multiorgan failure. Successful treatment included hemodialysis, red cell exchange transfusion, and therapeutic plasma exchange. Though SCD-related multiorgan failure and deferasirox-related hyperammonemia are reported, this case suggests multifactorial etiology including COVID-19-related hyperinflammation. Awareness of potential hepatic and systemic complications, and consideration for exchange transfusion and therapeutic plasma exchange, may reduce severity of COVID-19 sequelae in SCD.
\end{abstract}

\section{Introduction}

SARS-CoV-2 infection causing COVID-19 in sickle cell disease (SCD) is still being described. Severe COVID19 includes a broad spectrum of illness, including excessive pro-inflammatory cytokine release with multisystem hyperinflammation, vasculitis, and coagulopathy ${ }^{1}$; many die from ARDS, thromboembolic complications, or multiorgan failure ${ }^{2,3,4}$. Although acute chest syndrome and vaso-occlusive pain crisis (VOC) in SCD patients with COVID-19 have been reported ${ }^{5}$, we present a unique case of hepatic encephalopathy, hyperammonemia, and multisystem inflammation with multiorgan failure in a SCD patient following COVID-19.

\section{Case Description}

19-year-old female with SCD (S/ $\beta^{0}$ thalassemia-type), chronic pain syndrome, history of leg ulcers and hepatic crisis, on monthly transfusions with iron overload (average ferritin $3465 \mathrm{ng} / \mathrm{mL}$ over prior year) requiring deferasirox (DFX) chelation, was hospitalized for VOC when COVID-19 was diagnosed by positive SARSCoV-2 PCR. Her pain improved, she started hydroxychloroquine, and was discharged three days later. She returned after two days with nausea, emesis, dehydration, and transient AST/ALT elevation, presumed due to hydroxychloroquine, (thereafter discontinued). She developed acute chest syndrome with fever, decreased hemoglobin, but no hypoxemia, managed with simple red cell transfusion and antibiotics, and was discharged a few days later.

Four weeks later she was re-hospitalized with persistent anorexia and fatigue, dyspnea, and diffuse worsening pain, but with headache and abdominal pain not typical for her usual VOC. She received morphine and ketorolac and continued home medications including DFX. Repeat SARS-CoV2 nasal PCR was negative twice this hospitalization. On hospital day three, she developed fever $38.9^{\circ} \mathrm{C}$ for which blood culture was obtained and ceftriaxone administered. Five hours later she became acutely disoriented and agitated, without any 
focal neurologic deficit, and was transferred to the ICU. Laboratory evaluation revealed elevated ammonia of $350 \mathrm{mmol} / \mathrm{L}$, lactate $7.9 \mathrm{mmol} / \mathrm{L}$, AST 55 unit/L, ALT 29 unit/L, ALP 92 unit/L, TB $4.2 \mathrm{mg} / \mathrm{dL}, \mathrm{Cr}$ $1.3 \mathrm{mg} / \mathrm{dL}$ (baseline 0.7), normal albumin, mildly increased PT $(17.8 \mathrm{sec})$, and elevated CRP $(6.7 \mathrm{mg} / \mathrm{dL})$ (Figures 1, 2). CT Head and non-contrast MRI brain were normal; abdominal ultrasound showed isolated cholelithiasis and normal hepatoportal blood flow. She started lactulose and neomycin for hyperammonemia, and DFX was held due to possible toxicity. Her clinical encephalopathy progressed, with EEG consistent with severe diffuse cerebral dysfunction; she was then intubated for declining neurologic status. She underwent hemodialysis for progressive encephalopathy with persistent hyperammonemia, with improved ammonia from $369 \mathrm{mmol} / \mathrm{L}$ to $153 \mathrm{mmol} / \mathrm{L}$ (Figure 1). She developed elevated transaminases (Figure 1) and worsened synthetic liver function (PT $23.1 \mathrm{sec}$, bilirubin $10.7 \mathrm{mg} / \mathrm{dL}$ ), shock requiring vasoactive support, increased creatinine to $1.49 \mathrm{mg} / \mathrm{dL}$, and thrombocytopenia (platelet 95,000) with coagulopathy (normal fibrinogen $249 \mathrm{mg} / \mathrm{dL}$ but elevated D-dimer $4.4 \mathrm{mcg} / \mathrm{mL}$ - Figure 2), consistent with multiorgan failure syndrome (MOFS). Because of this, erythrocytapheresis (red blood cell exchange transfusion, RBCX) was performed (hemoglobin S pre-/post-RBCX $29 \%$ and 11\% respectively). Despite RBCX, CRP increased further to 15.1 $\mathrm{mg} / \mathrm{dL}$, with persistent thrombocytopenia and fever, so therapeutic plasma exchange (TPE) was performed for cytokine clearance and thrombocytopenia-associated MOFS ${ }^{6,7}$. Following TPE, AST, ALT, PT, and ammonia all normalized (Figure 1). CRP decreased to $2.1 \mathrm{mg} / \mathrm{dL}$, with resolved fever, and neurologic exam revealing purposeful movements. However, repeat brain MRI noted interval bilateral thalamic T2 hyperintensity, concerning for ischemia or localized cytotoxic edema from hepatic encephalopathy. She also developed a nonocclusive catheter-related left common femoral vein thrombus, so enoxaparin anticoagulation was started (prophylactic anticoagulation not started earlier due to coagulopathy). Five days after TPE, she returned to neurologic baseline without any deficits. She was discharged on hospital day 15 with residual but improved pain, improved D-dimer and CRP (Figure 2), and normal liver function tests. On outpatient follow-up one week later, her CRP normalized.

\section{Discussion}

MOFS is a known catastrophic complication of SCD; however, aspects of this case of MOFS with severe encephalopathy secondary to acute liver failure with hyperammonemia, acute kidney injury, and neurologic insult present a diagnostic enigma. MOFS in SCD typically evolves from VOC progressing to fever, altered sensorium, significant rapid drop in hemoglobin and platelets, and acute failure of at least two of the following organs: liver, kidney, or lungs; it is often fatal without prompt $\mathrm{RBCX}^{8,9}$. While she had some classic features of SCD-related MOFS, hyperammonemia is not typical, and her anemia and thrombocytopenia were mild. While her low baseline hemoglobin $\mathrm{S}(<30 \%)$ is not fully protective, it reduces the likelihood that SCD alone is the culprit.

Her history of acute and chronic liver issues - poorly-controlled iron overload, history of hepatic crisis ${ }^{10,11}$, potential toxicity from $\mathrm{DFX}^{12,13}$ and/or hydroxychloroquine - certainly predispose to hepatic injury ${ }^{14}$; however, these are unlikely the sole cause. In the differential for her acute liver dysfunction and hyperbilirubinemia was acute SCD hepatopathy, a range of conditions including acute hepatic crisis, hepatic sequestration, or acute intra-hepatic cholestasis. Though her baseline hemoglobin S was kept low by chronic transfusion therapy, evidence suggests that low hemoglobin S may not be fully protective against SCD hepatopathy ${ }^{10,7}$. However, involvement of other organs and unusual presentation of hyperammonemia preceding AST/ALT increase argue against SCD hepatopathy alone. DFX-induced liver failure and hyperammonemia are reported though poorly understood; however additional findings of MOFS with multisystem hyperinflammatory disorder also suggest a pathophysiology beyond DFX toxicity alone ${ }^{8,9}$.

While SARS-CoV-2 PCR was negative immediately prior to developing MOFS, the short duration from initial diagnosis to MOFS is suspicious. Severe COVID-19 disease was initially described as primarily ARDS; however, multiple organ failure, diffuse thromboembolic disease, and hyperinflammatory response have more recently been reported. Overproduction of early pro-inflammatory cytokines, including TNF- $\alpha$ and IL-6, may occur, leading to hyperinflammation with increased vascular permeability, vasculitis, and multiorgan failure $^{1}$. One such presentation has been described as multisystem inflammatory syndrome in children 
(MIS-C), a rare syndrome similar to Kawasaki Disease occurring one to six weeks following COVID-19 infection $^{15-17}$. MIS-C has greater gastrointestinal symptoms, lesser respiratory symptoms, and greater risk for severe manifestations of toxic-shock-syndrome, cardiac involvement (coronary aneurysms, myocarditis, heart failure), and macrophage activation syndrome ${ }^{18,19}$.

COVID-19-related coagulopathy is also recognized, with severe cases showing increased D-dimer, initial increase with late decrease in fibrinogen, disseminated intravascular coagulation ${ }^{20}$ or other thrombotic complications $^{18}$. Among other COVID-19 manifestations, gastrointestinal symptoms and/or abnormal liver function tests are reported ${ }^{12,13,21}$. Though this patient initially presented with VOC and respiratory symptoms of COVID-19, her acute chest syndrome was mild, without progression to pneumonitis, pulmonary edema, or ARDS; later manifestations were abdominal pain, hyperammonemia, liver failure, acute kidney injury, and systemic inflammation. While this case is not consistent with MIS-C, it demonstrates that postCOVID-19 inflammation may include a spectrum of manifestations occurring after initial infection. Further observation supporting COVID-19-related multisystem hyperinflammatory disorder is her rapid improvement following TPE, observed in other COVID-19 patients ${ }^{22,23}$.

The pathophysiologic mechanism for this patient's clinical course is likely multifactorial, with COVID19-related multisystem hyperinflammatory disorder likely triggering SCD-related MOFS and DFX-induced hepatic injury with hyperammonemia in a patient with preceding liver disease. This novel case of MOFS with predominant hepatic involvement may represent another possible COVID-19 manifestation in SCD, preexisting liver disease, DFX therapy, or others without these conditions. We recommend monitoring liver function, coagulation profile, and ammonia levels in high risk COVID-19 patients with underlying liver disease (SCD or other causes). Hepatotoxic drugs like DFX may need to be held. Lastly, RBCX and TPE should be considered in SCD patients with MOFS and multisystem hyperinflammatory disorder secondary to current or recent COVID-19.

Conflict of Interest: The authors have no conflicts of interest to disclose.

Financial Disclosure: The authors have no financial relationships relevant to this article to disclose.

\section{References:}

1. Jose RJ, Manuel A. COVID-19 cytokine storm: the interplay between inflammation and coagulation. The Lancet Respiratory . April 2020:1-2. doi:10.1016/S2213-2600(20)30216-2.

2. Zhou F, Yu T, Du R, et al. Clinical course and risk factors for mortality of adult inpatients with COVID-19 in Wuhan, China: a retrospective cohort study. The Lancet . 2020;395(10229):1054-1062. doi:10.1016/S01406736(20)30566-3.

3. Garg S, Kim L, Whitaker M, O'Halloran A, Cummings C, Holstein R. MMWR - Hospitalization Rates and Characteristics of Patients Hospitalized with Laboratory-Confirmed Coronavirus Disease 2019 - COVIDNET, 14 States, March 1-30, 2020. CDC MMWR . 2020;69(15):458-464.

4. Zhao D, Yao F, Wang L, Zheng L, Gao Y, Ye J. A comparative study on the clinical features of COVID-19 pneumonia to other pneumonias. Clin Infect Dis . March 2020:1-21. doi:10.1093/cid/ciaa247/5803302.

5. Nur E, Gaartman AE, Tuijn CFJ, Tang MW, Biemond BJ. Vaso-occlusive crisis and acute chest syndrome in sickle cell disease due to 2019 novel coronavirus disease (COVID-19). Am J Hematol . April 2020. doi:10.1002/ajh.25821.

6. Zaidi GZ, Rosentsveyg JA, Fomani KF, Louie JP, Koenig SJ. Reversal of Severe Multiorgan Failure Associated with Sickle Cell Crisis Using Plasma Exchange: A Case Series. J Intensive Care Med . 2019;35(2):140148. doi:10.1177/0885066619874041.

7. Fortenberry JD, Nguyen T, Grunwell JR, et al. Therapeutic Plasma Exchange in Children with Thrombocytopenia-Associated Multiple Organ Failure. Critical Care Medicine . 2019;47(3):e173-e181. doi:10.1097/CCM.000000000003559. 
8. McCollom JW, Dublis SA, Hoogeboom J, Doyle A, Templin J. Catastrophic Multi-Organ Failure with Bone Marrow Necrosis in a Sickle Cell Beta Plus Thalassemia Patient. Blood . 2014;124(21):4942-4942. doi:10.1182/blood.V124.21.4942.4942.

9. Kim HC. Red cell exchange: special focus on sickle cell disease.Hematology . 2014;2014(1):450-456. doi:10.1182/asheducation-2014.1.450.

10. Banerjee S, Owen C, Chopra S. Sickle cell hepatopathy.Hepatology . 2001;33(5):1021-1028. doi:10.1053/jhep.2001.24114.

11. Allali S, de Montalembert M, Brousse V, et al. Hepatobiliary Complications in Children with Sickle Cell Disease: A Retrospective Review of Medical Records from 616 Patients. JCM . 2019;8(9):1481-1496. doi:10.3390/jcm8091481.

12. Menaker N, Halligan K, Shur N, Paige J, Hickling M. Acute Liver Failure During Deferasirox Chelation: A Toxicity Worth Considering.J Pediatr Hematol Oncol . 2017;39(3):217-222.

13. Ramaswami A, Rosen DJ, Chu J, Wistinghausen B, Arnon R. Fulminant Liver Failure in a Child With b-Thalassemia on Deferasirox: A Case Report. J Pediatr Hematol Oncol . 2017;39(3):235-237.

14. Shah R, Taborda C, Chawla S. Acute and chronic hepatobiliary manifestations of sickle cell disease: A review. WJGP . 2017;8(3):108-116. doi:10.4291/wjgp.v8.i3.108.

15. Chiotos K, Bassiri H, Behrens EM, Blatz AM, Chang J. Multisystem Inflammatory Syndrome in Children during the COVID-19 pandemic: a case series. The Journal of the Pediatric Infectious Diseases Society . May 2020:1-25. doi:10.1093/jpids/piaa069/5848127.

16. Verdoni L, Mazza A, Gervasoni A, et al. An outbreak of severe Kawasaki-like disease at the Italian epicentre of the SARS-CoV-2 epidemic: an observational cohort study. The Lancet . 2020;395(10239):17711778. doi:10.1016/S0140-6736(20)31103-X.

17. Whittaker E, Bamford A, Kenny J, et al. Clinical Characteristics of 58 Children With a Pediatric Inflammatory Multisystem Syndrome Temporally Associated With SARS-CoV-2. JAMA . June 2020:1-11. doi:10.1001/jama.2020.10369.

18. Belhadjer Z, Méot M, Bajolle F, et al. Acute heart failure in multisystem inflammatory syndrome in children (MIS-C) in the context of global SARS-CoV-2 pandemic. Circulation . 2020;382:1370-22. doi:10.1161/CIRCULATIONAHA.120.048360.

19. Toubiana J, Poirault C, Corsia A, et al. Kawasaki-like multisystem inflammatory syndrome in children during the covid-19 pandemic in Paris, France: prospective observational study. BMJ . 2020;105:m2094m2097. doi:10.1136/bmj.m2094.

20. Connors JM, Levy JH. COVID-19 and its implications for thrombosis and anticoagulation. Blood . April 2020:1-21. doi:10.1182/blood.2020006000.

21. Cheung KS, Hung IF, Chan PP, et al. Gastrointestinal Manifestations of SARS-CoV-2 Infection and Virus Load in Fecal Samples from the Hong Kong Cohort and Systematic Review and Metaanalysis. Gastroenterology . March 2020. doi:10.1053/j.gastro.2020.03.065.

22. Ma J, Xia P, Zhou Y, et al. Potential effect of blood purification therapy in reducing cytokine storm as a late complication of critically ill COVID-19. Clinical Immunology . 2020;214. doi:10.1016/j.clim.2020.108408.

23. Keith P, Day M, Perkins L, Lou Moyer, Hewitt K, Wells A. A novel treatment approach to the novel coronavirus: an argument for the use of therapeutic plasma exchange for fulminant COVID-19. Critical Care . 2020;24(1):128-131. doi:10.1186/s13054-020-2836-4.

\section{Legends:}


Figure 1. Liver function tests and ammonia levels during hospitalization (AST: aspartate aminotransferase; ALT: alanine aminotransferase; RBCX: red blood cell exchange transfusion; TPE: therapeutic plasma exchange)

Figure 2. CRP and D-dimer levels during hospitalization (CRP: C-reactive protein; RBCX: red blood cell exchange transfusion; TPE: therapeutic plasma exchange)

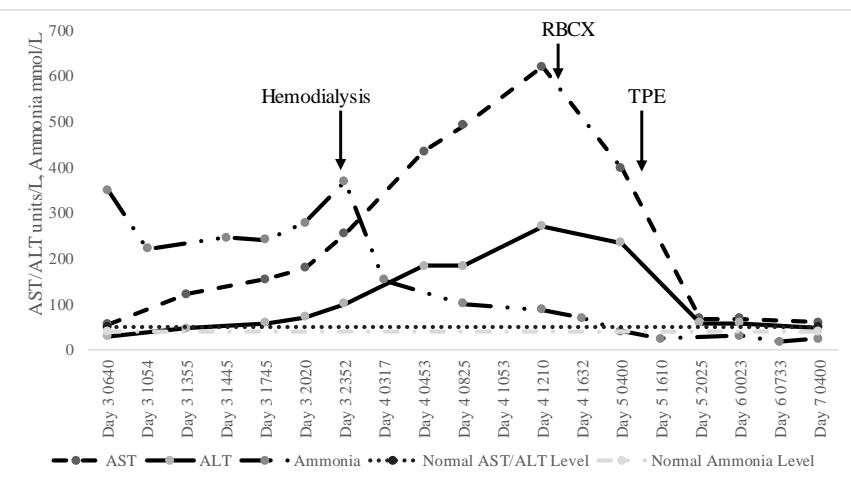




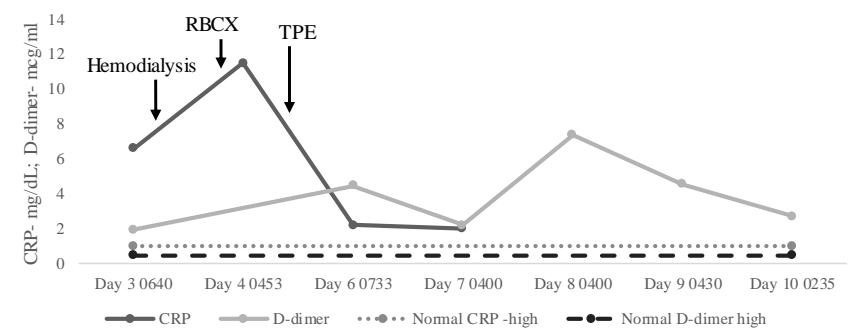

